of something like 1800 entries (which the author states constitute only half his collection of references) is in itself enough to make this an indespensable work of reference. With the companion Volume 1 (of which a slightly revised third edition has now appeared) it forms a contribution far beyond the range of ordinary textbooks, suitable for reference and for serious students.

\title{
HAROLd HotelLING
}

The methods of plane projective geometry based on the use of general homogeneous coordinates. By E. A. Maxwell. Cambridge University Press, $1946.19+230$ pages. $\$ 2.75$.

This is an introductory treatise on algebraic projective geometry. As the author states in the preface, "It is written as a study of methods and not as a catalogue of theorems." Synthetic methods are used occasionally when deemed preferable. Although it is stated in the introduction, "No knowledge of geometry is assumed explicitly," it is implied that the student should have had plane analytical geometry, including the properties of conic sections, before beginning the study of this book.

The first ten chapters deal with non-metric concepts. The principal topics and the order in which they occur are: homogeneous coordinates, equations of lines, duality, one-to-one correspondence cross ratio, conics treated parametrically, projective properties of conics, the quadrangle and its dual, the generation of conics by pairs of projective pencils or ranges, pencils of conics, harmonic properties, reciprocation, poristic systems, theorems of Pascal and Brianchon.

In the last two chapters, eleven and twelve, relations between projective and Euclidean geometry are studied both algebraically and synthetically.

The book is well written; the explanations are clear and ample. The printing is excellent and the formulas well displayed. The caption of each section describes pithily the contents. The book has an excellent index and a very detailed table of contents. The total absence of figures, however, is to be deplored; illustrative drawings are especially helpful to a beginner in projective geometry.

The many excellent and carefully chosen problems constitute one of the best features of the book. These problems are chiefly taken from various examinations. At the end of each problem is a symbol referring to the source given in the preface. Answers to the more difficult problems are given in the back of the book.

T. R. HOLLCROFT 